\title{
Knowledge And Attitude on Electro Convulsive Therapy Among Primary Care Givers
}

\author{
*Jeeva Christopher, **Manoranjitham .S \\ ${ }^{I} \operatorname{M.Sc}(N)$, M.sc(Psy), PGD(Guidance \& Counseling ) \\ ${ }^{2} S, M . S c(N)$., $P h . D$
}

\begin{abstract}
:
Background \& objectives: Electro Convulsive Therapy (ECT) is used frequently in developing countries, but investigations of care givers Knowledge and Attitude towards ECT are rare. The present study thus attempted a comprehensive examination of care givers knowledge, and attitudes towards ECT.

Methods: Descriptive study was employed to study the level of knowledge and attitude towards ECT among the primary care givers of patients who are receivingECT. A sample of 30 consecutive primary care givers of patients who received ECT was recruited for the study.

Results: Care Givers mainly middle aged, males, Hindus, educated up to secondary level, doing unskilled works and were parents. There was significant relationship between adequate knowledge and the positive attitude.(p=0.001).There is a significant relationship between the Number of Hospitalization with Level of Knowledge $(p=0.018)$, Knowledge level and Religion $(\boldsymbol{p}=\mathbf{0 . 0 4 7 )}$, knowledge level andthe number of hospitalization. $(\boldsymbol{p}=\mathbf{0 . 0 0 9})$. There is a positive correlation $(r=0.576)$ between the knowledge and attitude towardsECT; primary care givers, who have adequate knowledge aboutECT, have a positive attitude towards it. On the other hand those who don't know much about ECT have an ambivalent and negative attitude towardsECT.
\end{abstract}

Interpretation \& conclusions: The results endorse the notion that the knowledge and attitude towards ECT among primary care givers was inadequate and majority of them have negative attitude.

Keywords: Primary care giver, Electro Convulsive Therapy, Knowledge and Attitude.

\section{Introduction}

Electro convulsive therapy, a procedure which produces a deliberate, artificially induced grandmal seizure has been widely used to treat mentally ill patients since its introduction in 1938. Seizure activity is the therapeutic aspect of this form of treatment, but it may be accompanied by untoward physiological consequences(Carney .S, Geddes. J2003).

$E C T$ treatment has been associated with short term memory loss \& difficulty in learning. People may have trouble in memory that occurred in the days before the treatment or earlier. The most common side effects of $E C T$ on the day of treatment include nausea, headache, fatigue, confusion and slight memory loss, which may last minutes to hours (American Psychiatric Association, 2010).

$E C T$ is among the safest and most effective treatments available for depression. With electrodes are placed on the patients scalp and finely controlled electric current is applied while the patient is under General Anesthesia. The current causes a brief seizure in the brain. $E C T$ is one of the fastest ways to relieve symptoms in severely depressed or suicidal patients. It's also very effective for patients who suffer from mania or other mental illnesses. (Keller 2015).

Treatment with ECT is highly effective and works rapidly when compared with other psychiatric treatments .While antidepressants may take between two or three months to have a complete effect. Some people who are treated with ECT may begin to feel better within one week of beginning of their treatment. ECT is often used in patients with severe suicidal thoughts or behaviors as it works significantly faster than other treatments to decrease these distressing symptoms. It can also work to reverse complex symptoms of mania that may have been treatment resistant. ECT can also be used for catatonic symptoms.(Jacob Freedman 2012).

Rajgopal \&Chakrabarti (2010) conducted a study to assess the caregiver's knowledge, experience and attitudes among 153 relatives of 77 patients. Most of the patients were largely unaware of the procedure. Though most did not find the experience of ECT upsetting, considerable proportions expressed dissatisfaction with aspects such as informed consent, fear of treatment and memory impairment. Although patients were mostly positive aboutECT, ambivalent attitudes were also common, but clearly negative views were rare. Relatives were significantly likely to be more aware, more satisfied with the experience and have more favorable attitudes towardsECT, than patients.

Alaa el din (2013) conducted a study in Egypt to assess the knowledge and attitude about ECT among care givers of patients with psychiatric disorders among 450 samples. The result was $50.4 \%$ of the participants had 
not received information aboutECT. High percentage of participants had correct knowledge and positive attitudes towardsECT. The main significant factor was affecting their knowledge and attitude was the previous experience of their patients withECT.

A study was conducted byGrover\&Chakrabarti(2014) in PGIMER, with 100 patients and their relatives who hadECT; primary care givers were better informed and more positive about ECTthan patients, but the differences between two groups were not significant. Patients obtained information, mostly from media $(44 \%)$, doctors $(23 \%)$, and from personal experiences $(13 \%)$. On the other hand, relatives obtained information almost equally from media (26\%), doctors $(27 \%)$, and experience of friends or relatives (28\%). No significant difference was observed in knowledge and attitude in patients who had obtained their facts from doctors and from other sources. Among relatives, those who had obtained their information from doctors were better informed than those who had obtained so from other sources. Since patients and relatives have poor knowledge and negative attitude towardECT, medical professionals should impart proper information about ECT to patients and relatives to increase the acceptability of this treatment.

\section{Objectives}

1. To assess the knowledge and attitude on ECT among the primary care givers.

2. To determine the association between knowledge \& attitude on ECT and the clinical variables (patients) and demographic variables of the primary care givers.

\section{Hypothesis:}

- $\quad$ There is significant relationship between knowledge and attitude of Electro Convulsive Therapy with selected demographic variables.

\section{Methods}

Descriptive study was employed to study the level of knowledge and attitude towards ECT among the primary care givers of patients who are receiving ECT . The study was conducted in a tertiary teaching hospital in south India. The population comprises of the primary care givers of psychiatric patients who receivedECT. Those who provided informed consent were considered as the sample. A sample of 30 consecutive primary care givers of patients who were receiving ECT was recruited for the study. The data was collected during a single session for a period of 12 weeks between $7.30 \mathrm{am}$ to $12.30 \mathrm{pm}$ and $4 \mathrm{pm}$ to $7 \mathrm{pm}$. Necessary permission to conduct the study was requested and obtained from the head of the psychiatry and psychiatric nursing departments respectively. Oral and written consents were obtained from the subjects prior to the data collection and confidentiality with the information was ensured throughout the study.

\section{Inclusion criteria}

- Primary care givers of patients who received ECTand attended at least 3 ECT's along with the patient.

- Primary care givers who are above the age of 20 years

\section{Exclusion criteria}

- Primary care givers who are unable to communicate in English, Tamil and Hindi.

- Primary care givers who are suffering with chronic physical or psychiatric illness.

- Primary care givers who are unwilling to participate in this study.

There are 3 parts of assessment for this study.

\section{Instruments}

Part l--------socio demographic and clinical variables

Section A-socio demographic data of the patient includes age, gender, religion, marital status, education, occupation, type of family and monthly income.

Clinical variable of the patient-includes duration of illness, number of hospitalizations, previousECT, diagnosis of the patient and number ofECT.

Section B----socio demographic data of the primary care giver includes age, gender, religion, marital status, education, occupation, type of family, monthly income, relationship with the patient and duration of stay with the patient.

The knowledge and attitude questionnaire is developed by Freeman and Kendell in 1980 and modified by Tang et al in 2002 and validated among large population.

Part ll--rating scale for assessing knowledge on ECT.

Knowledge was assessed using a 30-item questionnaire. Each item had a correct, an incorrect and a 'don't know' response. Incorrect and 'don't know' responses were clubbed together, because both signified that the 
participant was unaware. The knowledge level of the primary care giver towards ECT was categorized as follows: Adequate-more than 50\% \&Inadequate---less than $50 \%$.

Part III--rating scale for assessing attitude towards ECT.

These were assessed using a 16-item questionnaire. Each item had 3 alternatives based on which responses were categorized into positive, negative or ambivalent attitudes. The questionnaire covered areas of efficacy, safety, frequency of use, indications for, mode of action, side effects and practical aspects ofadministration.

\section{Results}

The Statistical Software SPSS for windows (version17.0) was employed for the analysis of data.The subjects were selected using consecutive sampling technique. Descriptive statistics was used to present the frequency and percentage of demographic variables. Chi square was used to infer the study findings. The findings are presented as follows:

Section A: Description of socio-demographic \&clinical variables of patients and socio-demographic variables of primary care givers.

Section B:Knowledge and attitude on ECT among the primary care givers of patientswho receivedECTSection C: Association of socio-demographic variables of primary care giver with knowledge and attitude onECT.

Section D: Correlation between knowledge and attitude of ECT among the primary care givers.

\section{Section B figure 1: level of knowledge on ECTamong the primary care givers of patient who} receivedECT.

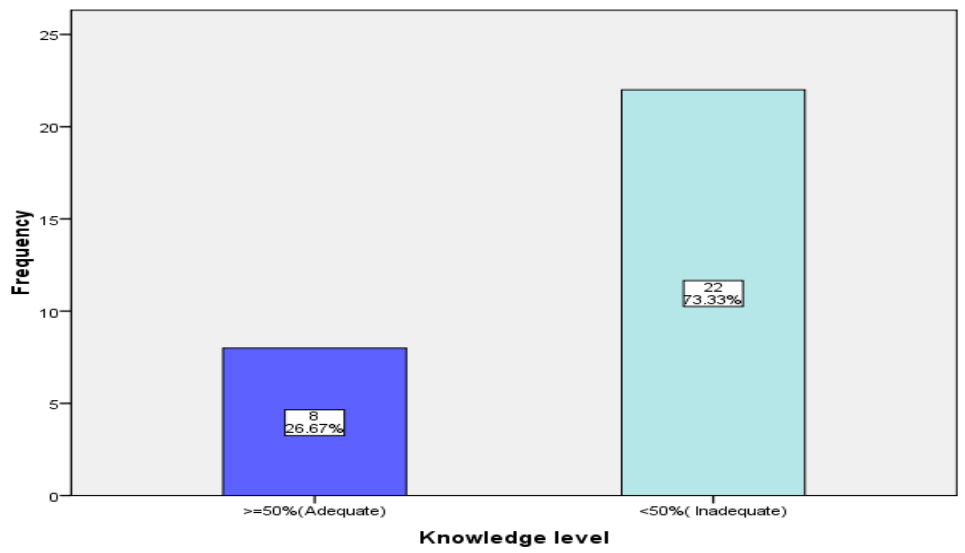

Figure 1: The above histogram reveals that majority of the care givers (73.3\%) have inadequate knowledge; and only $26.7 \%$ of them have adequate knowledge towardsECT.

Section B figure 2: Level of attitude towards ECT among the primary care givers of patient who receivedECT.

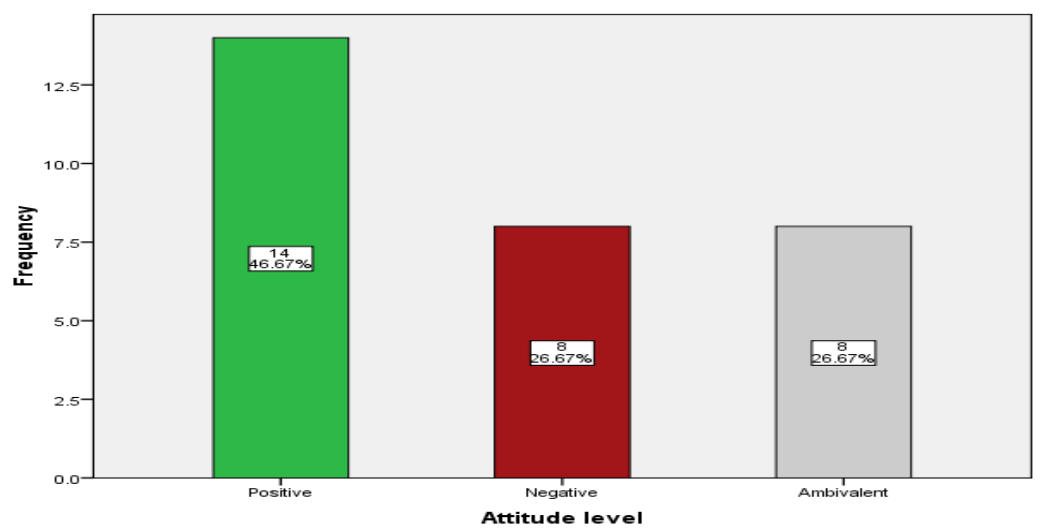

Figure 2: The above histogram reveals that $46.7 \%$ of them have positive attitude; $26.7 \%$ of them have ambivalent; and $26.6 \%$ have negative attitude towardsECT

Section D Figure 3: Correlation between knowledge and attitude among primary care givers 


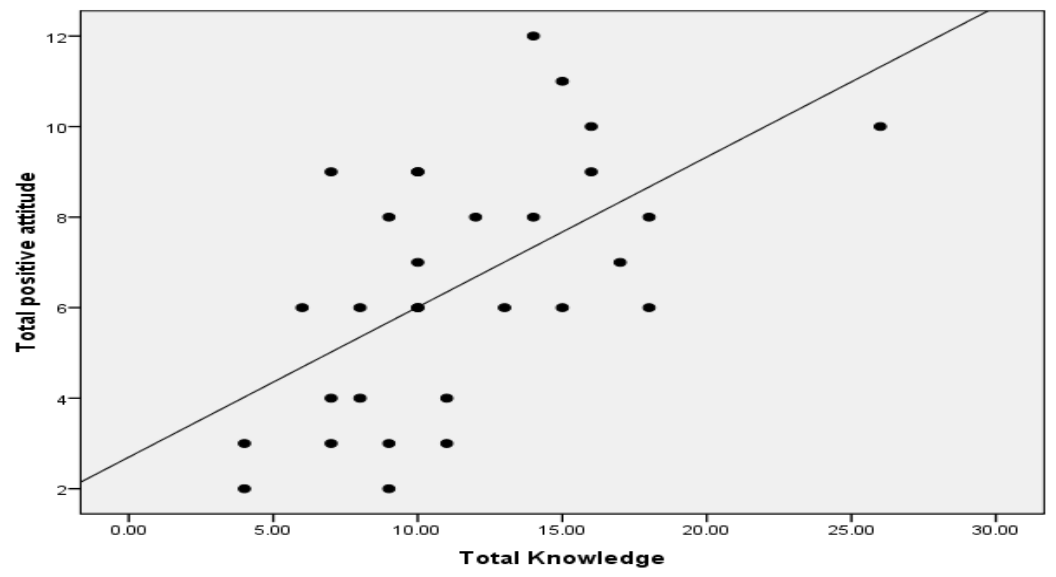

$\mathbf{P}=0.091 \quad R=0.576$

Figure 3: Theabove scatter plot reveals that there is a positive correlation $(r=\mathbf{0 . 5 7 6})$ between the knowledge and attitude towards ECT; primary care givers, who have adequate knowledge aboutECT, have a positive attitude towards it.

\section{Discussion}

This descriptive study was conducted with the objective of assessing the level of knowledge and attitude among the care givers of patient who received ECT, 30 consecutive samples were interviewed and data were collected.

The first objective of the studywas to assess the knowledge and attitude on ECT among the primary care givers. Thestudy shows $8(26.7 \%)$ has adequate knowledge, 22(73.3\%) has inadequate knowledge regarding ECT also the study shows $46.7 \%$ of them have positive attitude; $26.7 \%$ of them have ambivalent: and $26.6 \%$ have negative attitude towards ECT. This study was supported by the findings of the study conducted byGrover \&Chakrabarti(2014) in PGIMER, with 100 patients and their relatives who hadECT; majority of the patients and their primary care givers were unaware of the basic facts aboutECT. Previous experience ofECT did not have any major impact in knowledge and attitude in both patients and primary care givers. No significant difference was observed in knowledge and attitude in patients who had obtained their facts from doctors and from other sources.

The second objective of this study was to determine the association between demographic variables and knowledge \& attitude aboutECT.It was found that majority of the care givers have inadequate knowledge in all the age group, gender, religion. Majority (57.1) of the graduates has adequate knowledge and the illiterates have $100 \%$ inadequate knowledge. Professionals have (100\%) adequate knowledge. 50\% of the spouses and those who earn more than 5000/month has adequate knowledge towardsECT. The study result revealed that the care givers between the age group of 19-45 (75\%), male gender (52.2\%), Christians (66.7\%), graduates $(57.1 \%)$, professionals (100\%), earning around $5000(60 \%)$, married $(75 \%)$ and children of the patients $(66.7 \%)$ show positive attitude. There is a significant relationship between the number of hospitalization with level of knowledge $(\mathrm{p}=\mathbf{0 . 0 1 8})$, knowledge level and religion $(\mathbf{p = 0 . 0 4 7})$. There was significant relationship between the adequate knowledge and the positive attitude. ( $\mathbf{p = 0 . 0 0 1 )}$.there is a positive correlation $(\mathrm{r}=\mathbf{0 . 5 7 6})$ between the knowledge and attitude towardsECT; primary care givers, who have adequate knowledge aboutECT, have a positive attitude towards it. On the other hand those who don't know much about ECT have an ambivalent and negative attitude towardsECT.

This study finding was supported by the findings of the study conducted by Rajkumar, Saravanan \&Jacob KS (2006): to assess the knowledge of 52 patients and care givers knowledge who received ECT before and after the course of the treatment. More than half of the relatives were not aware of the details of ECT even at the end of the course. This study was also supported by the findings of the study conducted by Amitava Dan, Sandeep Grover (2014), to assess the knowledge and attitude of patients and their relatives toward electro convulsive therapy. Majority of the patients and relatives were unaware of the basic facts aboutECT, informed consent, indications, effectiveness and side effects. Relatives were somewhat better informed and more positive about ECT than the patients, but differences between the two groups were not significant. Previous experience of ECT did not have any major impact in knowledge and attitude in both the groups. There is no significant relationship between knowledge and attitude with socio demographic data.

\section{Conclusion}

The findings of the study revealed that the primary care givers have inadequate knowledge and negative attitude towardsECT, hence it is recommended that health care professionals should impart proper 
information aboutECT to patients and their care givers to increase the acceptability ofECT.

The practices and instructions about ECT needs to be improved to enhance and help the family members to acquire good knowledge and positive attitude not only towards ECT but also to the treatment regimen. The psychiatric health nurses can assess the existing knowledge and attitude towards ECT and design health teaching accordingly. Health care team should be involved in conducting regular public awareness program related to ECT benefits, side effects, procedure, therapeutic effects and safety as well as about other alternative suitable treatment choices. Educating the patients and care givers onECT should be intensified.

\section{References}

[1]. Carney s, Geddes j. Electroconvulsive therapy. Br Med J2003; 326: 1343-4

[2]. Kellner c. Unipolar major depression in adults: Indications for and efficacy of electroconvulsive therapy (ECT). Http://www.uptodate.com/home. Accessed Aug. 26, 2015.

[3]. Freeman CPL, Kendell RE. Ect: Patients' experiences and attitudes. Br J Psychiatry. 1980; 137:8-16.

[4]. Chakrabarti S, Grover S, Rajagopal r. 6. Perceptions and awareness ofElectro Convulsive Therapy among patients and their families: A review ofthe research from developing countries. J ECT2010; 26: 317-22.

[5]. Alaa El Din,Khaled A. Mohamed, Assessment of knowledge and attitude about Electro Convulsive Therapy among care givers of patients with different psychiatric disorders, Middle East Current Psychiatry 2013 20(4):205-215

[6]. Sandeep Grover \&Subho Chakrabarti; Indian J Psychol Med.2014 36(3): 264-269.

[7]. American Psychiatric Association. Practice guidelines forthe treatment of patients with major depressive disorder. Am J Psychiatry, 2010; 167(10): 69

[8]. Rajkumar AP, Saravanan B, Jacob KS. Perspectives of patients 24. And relatives aboutElectro Convulsive Therapy: A Qualitative study fromVellore, India. J ECT2006; 22: 253-8. 